\title{
Quantitative analysis of the elastic fiber in the tunica media at the carotid bifurcation
}

\author{
By \\ Yu KATO ${ }^{1}$, Tohru MIZUTANI ${ }^{1}$, Naruhito OTSUKA ${ }^{2}$, \\ Hiromitsu EZURE ${ }^{2}$, Yuriko INOUE ${ }^{2}$ \\ ${ }^{1}$ Department of Neurosurgery, Showa University School of Medicine, Tokyo 142-8555, Japan \\ ${ }^{2}$ Department of Anatomy, Showa University School of Medicine, Tokyo 142-8555, Japan
}

-Received for Publication, March 20, 2018 -

Key Words: Carotid artery, Bifurcation, Tunica media, Elastic fiber

\begin{abstract}
Summary: In this study, the results of our previously reported technique of quantitative analysis by using microscopic image analysis of tissue image slices to calculate the proportion of the area of the tunica media occupied by of elastic fibers was compared with Janzen et al.'s technique at the carotid bifurcation. This particularly analyzed the area of transition between the common carotid and the internal carotid, to observe the quantitative changes in elastic fiber content. The data obtained from our quantitative analysis of elastic fibers were clearly at variance with those obtained by counting the number of elastic fibers. The amount of elastic fibers in the tunica media (the elastic fiber ratio) decreased from the proximal carotid artery (the common carotid) to the bifurcation, then peaked in the internal carotid immediately after the bifurcation before declining again.
\end{abstract}

\section{Introduction}

Arteries are classified as either elastic or muscular depending on the histological characteristics of the tunica media ${ }^{1}$. In elastic arteries, the tunica media contains a large amount of elastic fibers, whereas in muscular arteries, smooth muscle predominates ${ }^{1)}$. The amounts of elastic fibers and smooth muscle, the main constituents of the tunica media, varies, and their ratio changes in the transitional zone between elastic arteries closer to the heart (proximal) and muscular arteries further away $(\text { distal })^{1}$. In the neck, the common carotid artery is classified as an elastic artery, and the internal carotid as a muscular artery ${ }^{1}$. The carotid bifurcation marks the junction between these two arterial types, and is an important point at which a quantitative change in the amount of elastic fibers in the tunica media is apparent.

The method that has most commonly been used for the quantitative analysis of the amount of elastic fibers in blood vessels is Janzen et al.'s method of counting the number of these fibers in the tunica media from tissue slices $^{2}$. Some previous quantitative analyses of carotid bifurcation have also used this method ${ }^{2}$,3). However, one limitation of this technique, as a method of quantitative analysis, is that it provides no information regarding the size (thickness) of the elastic fiber bundles, which may not accurately indicate the amount of elastic fibers at the carotid bifurcation.

In this study, the results of our previously reported technique of quantitative analysis by using microscopic image analysis of tissue image slices to calculate the proportion of the area of the tunica media occupied by of elastic fibers $\left.{ }^{4}\right)$ was compared with Janzen et al.'s technique $^{2)}$ at the carotid bifurcation. This particularly analyzed the area of transition between the common carotid and the internal carotid, to observe the quantitative changes in elastic fiber content. Histological observations of elastic fibers in the tunica media were also determined at this site.

\section{Materials and Methods}

\section{Study Materials}

Seven carotid bifurcations obtained from the neck of 4 cadavers (both sides, in some cases) for experimental 
dissection (mean age 77 years, range 63-89 years) were used for this study.

\section{Tissue Slice Preparation}

Arterial sections from the origin of the common carotid to the intracranial carotid were removed en bloc from the cadavers, which were preserved in $10 \%$ formalin. Viewed from the luminal face of the artery, the bifurcation was set as the point of origin ( 0 ), and a total of 15 blocks were prepared from circumferential cross-sections of the vessels at distances of $-20,-15,-10$, and $-5 \mathrm{~mm}$ proximal to this position (in the common carotid artery) and $+5,+10$, $+15,+20,+25,+30,+35,+40,+45$, and $+50 \mathrm{~mm}$ distal to this position (in the internal carotid artery) (Fig. 1). These were embedded in paraffin using an automatic embedding device (Sakura ETP-150CV, Sakura, Chikuma,

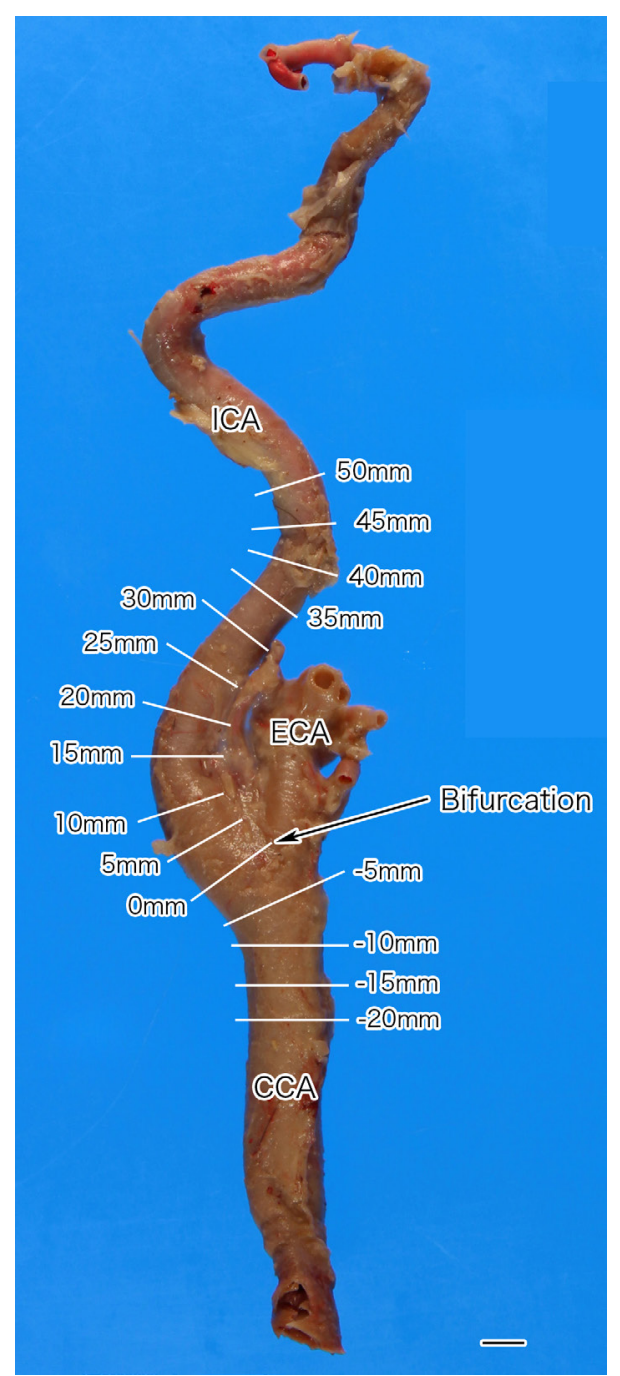

Fig. 1. Photograph of sampling position for the carotid artery CCA: common carotid artery; ICA: internal carotid artery; ECA: external carotid artery.
Japan), after which $5-\mu \mathrm{m}$ cross-sectional slices from all 15 positions were prepared using a sliding microtome and stained using Verhoeff-Van Gieson elastic staining (EVG staining) following a modification of Maeda's method.

\section{Quantitative Analysis of the Elastic Fiber Content of the} Tunica Media

\section{Elastic Fiber Ratio (Kato et al.'s method ${ }^{4)}$ )}

Four sampling areas of the entire thickness of the tunica media were selected arbitrarily at $90^{\circ}$ intervals on slices with EVG staining taken from each of the 15 proximal to distal positions $(-20 \mathrm{~mm}$ to $+50 \mathrm{~mm}$ ). Following the method reported by Kato et al. ${ }^{4)}$, the brightness level that identified only elastic fibers in the entire thickness of the tunica media at these positions was measured. Using this value as the threshold, the area occupied by elastic fibers as a proportion of the total area of the tunica media (the "elastic fiber ratio") was measured in each sampling area, and the mean values of four sampling areas at each position calculated (Fig. 1).

\section{Number of Elastic Fibers (Janzen et al.'s method ${ }^{2)}$ )}

Four sampling areas of the entire thickness of the tunica media were selected arbitrarily at $90^{\circ}$ intervals on slices with EVG staining with taken from each of the 15 proximal and distal positions $(-20 \mathrm{~mm}$ to $+50 \mathrm{~mm})$. Following the method reported by Janzen et al. ${ }^{2)}$, The number of elastic fibers existence in each sampling area was counted under 200x microscopy, and the mean values of four sampling areas at each position calculated (Fig. 1).

\section{Histological Observations of the Tunica Media}

The vascular wall, including the tunica media, was examined under light microscopy in slices with EVG staining taken from each of the 15 proximal to distal positions $(-20 \mathrm{~mm}$ to $+50 \mathrm{~mm})$.

\section{Results}

\section{Elastic Fiber Ratio}

The elastic fiber ratio decreased from $-20 \mathrm{~mm}$, reaching its minimum between $-15 \mathrm{~mm}$ and $0 \mathrm{~mm}$. It peaked between $+5 \mathrm{~mm}$ and $+10 \mathrm{~mm}$, after which it gradually declined (Fig. 3).

\section{Number of Elastic Fibers}

The number of elastic fibers in the tunica media increased from $-20 \mathrm{~mm}$, peaking at $-10 \mathrm{~mm}$ and $-5 \mathrm{~mm}$, after which it gradually declined (Fig. 4).

\section{Histological Observations of the Tunica Media}

From $-20 \mathrm{~mm}$ to $0 \mathrm{~mm}$, the tunica media contained a large amount of elastic fibers running parallel with the vascular wall as separate strands. The tunica media was thinner at $+5,+10,+15$, and $+20 \mathrm{~mm}$ compared with its 


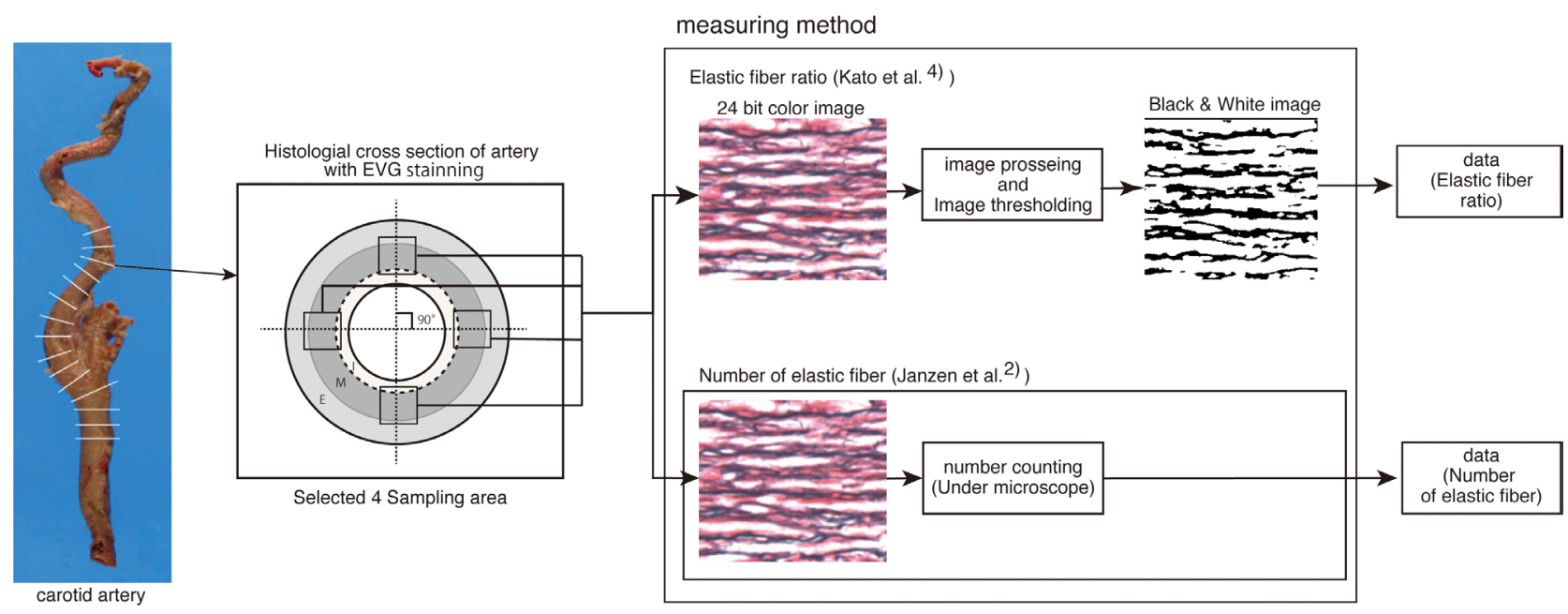

Fig. 2. Schematic explanation of measuring methods of the elastic fiber in this study E: tunica externa; I: tunica intima; M: tunica media

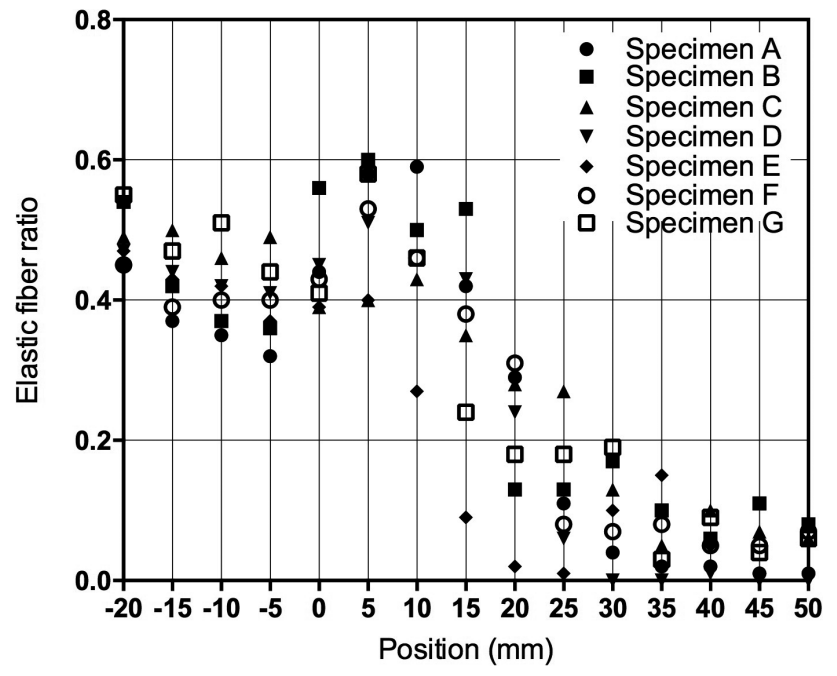

Fig. 3. Area ratio of elastic fiber in the tunica media in each sampling position.

thickness in both the common carotid $(-20,-15,-10$, and $-5 \mathrm{~mm})$ and the carotid bifurcation $(0 \mathrm{~mm})$. At +5 and $+10 \mathrm{~mm}$, these particular elastic fibers were somewhat thicker than those in other positions, and were more densely packed compared with the common carotid and the carotid bifurcation. At $+15,+20$, and $+25 \mathrm{~mm}$ many of the elastic fibers were discontinuous, exhibiting an insular or punctate structure. At positions distal to $+25 \mathrm{~mm}$, the tunica media was thicker than it was in the proximal internal carotid and contained very few elastic fibers, which were present as insular or punctate structures (Fig. 5).

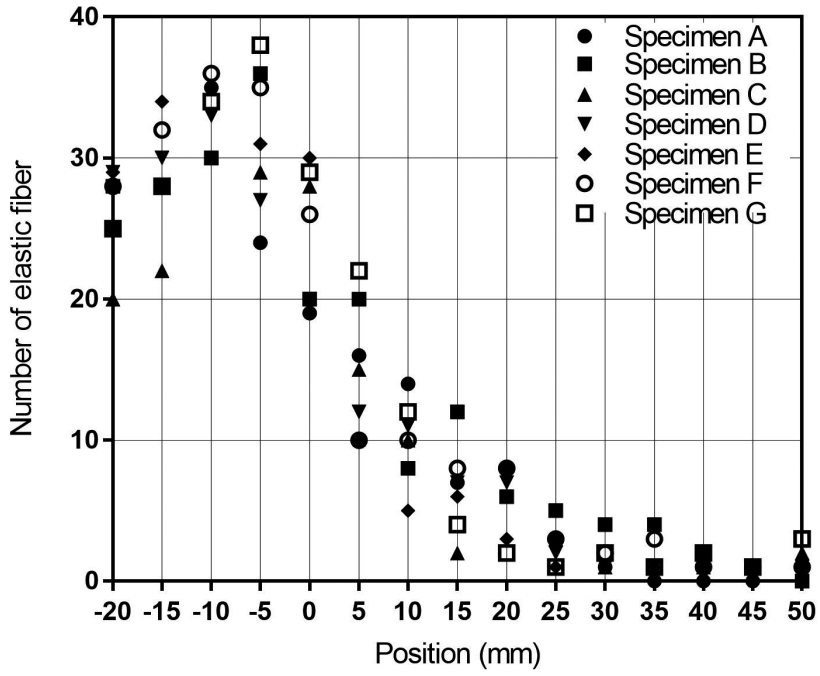

Fig. 4. Number of elastic fibers in the tunica media in each sampling position.

\section{Discussion}

In this study, the elastic fiber ratio was used, which is an index that we devised in a previous study), to carry out quantitative analysis regarding the amount of elastic fiber in the tunica media at the carotid bifurcation, alongside with microscopic histological observations of the elastic fibers in the tunica media. The two following points were established.

First, the data obtained from our quantitative analysis of elastic fibers were clearly at variance with those ob- 


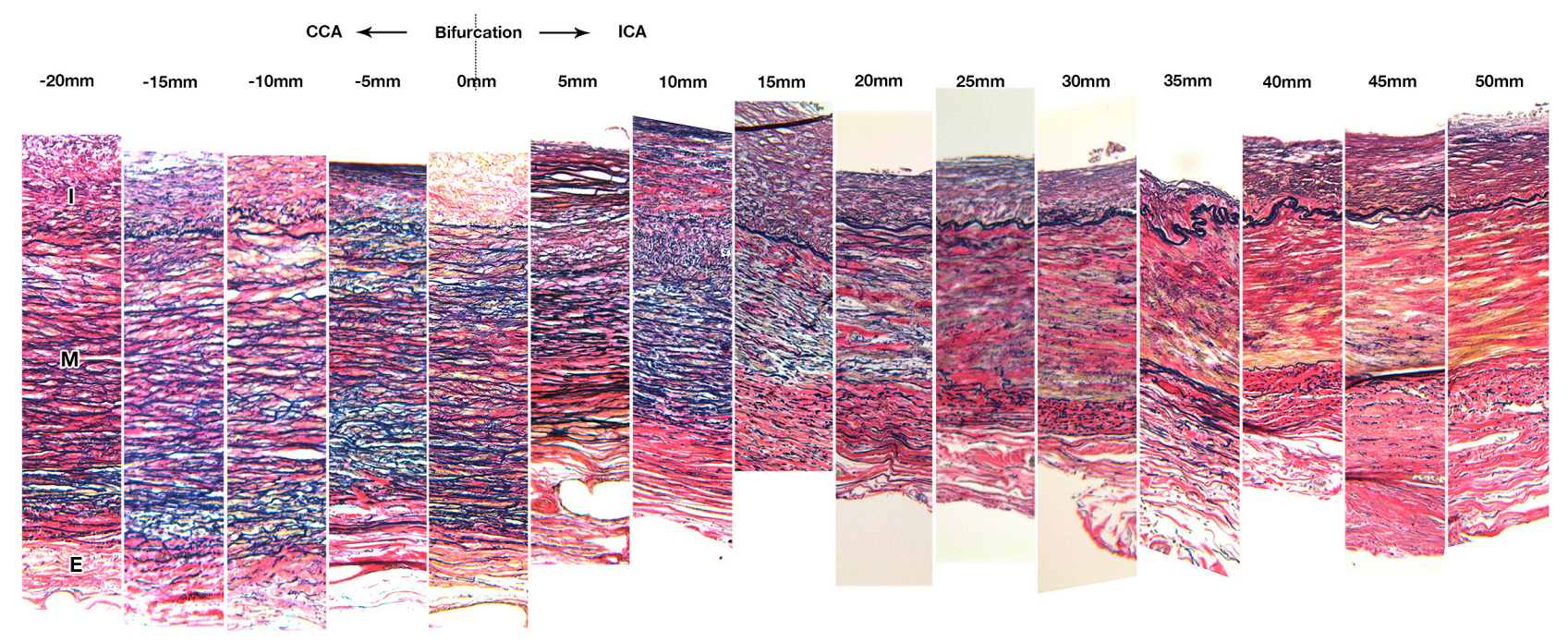

Fig. 5. Microscopic photographs of cross section of the carotid artery in each sampling position. E: tunica externa; I: tunica intima; M: tunica media

tained by counting the number of elastic fibers.

These differences were due to the different measurement methods employed. Most previous studies have used Janzen et al.'s method ${ }^{2)}$ of quantification by counting the number of elastic fibers in the tunica media on tissue slices. Unlike our elastic fiber ratio, this method of quantifying the amount of elastic fibers assumes that the shape and size (particularly the thickness) of the elastic fibers is constant, and that the thickness and area of the parts of the tunica media, in which the elastic fibers are being counted, are the same. However, previous studies have not paid any attention to these conditions. The histological observations in this study showed that the thickness of elastic fibers varied in different locations, being somewhat thicker in the area around the carotid bifurcation compared with the proximal carotid artery (the common carotid). Henmi ${ }^{5}$ ) also reported that the density, thickness, and tortuosity of elastic fibers differed in blood vessels in different locations, and that the size of the elastic fibers in the tunica media of the arterial wall also varied in different locations, which is in line with the findings of this study. This suggests that previous studies using the number of elastic fibers as a quantitative measure have failed to meet the necessary conditions, and that the use of this method to quantify the amount of elastic fiber in the carotid bifurcation may have limitations. Janzen et al.'s method ${ }^{2}$ is originally the arterial classification by the histological characteristics of the tunica media based on the number of elastic fiber in the tunica media ( $\geq 11$ : elastic artery; $\leq 5$ : muscular artery; 6-10: transitional), and was not enough as a means of quantitative analysis. Our elastic fiber ratio for the quantitative analysis of the amount of elastic fibers in the tunica media ${ }^{4}$ uses an image processing technique to identify only the elastic fibers and measure the proportion of the tunica media they occupy. This provides accurate measurements even when the size (thickness) of the elastic fibers varies by location such as carotid bifurcation, and also takes into account changes in the thickness and area of the tunica media at different position of artery by expressing the amount of elastic fibers as the proportion of the area of the tunica media. Therefore, it is considered that our method of quantifying the amount of elastic fibers in the tunica media as a proportion of the total area is more suitable than Janzen's method of counting the number of elastic fibers as a quantification method in present study at least.

Second, the amount of elastic fibers in the tunica media (the elastic fiber ratio) decreased from the proximal carotid artery (the common carotid) to the bifurcation, then peaked in the internal carotid immediately after the bifurcation before declining again (Fig. 3).

These quantitative changes in the amount of elastic fibers have not been previously reported. Arteries that contain blood under high pressure, such as those close to the heart, have high elastic fiber content ${ }^{1)}$, and studies have shown that the amount of elastic fibers in the tunica media increases when blood flow is experimentally increased $^{6}$. The amount is believed to be associated with perfusion-related stress. This suggests that the quantitative changes in the amount of elastic fibers seen in this study may represent the morphological adaptation of the tunica media in response to stress placed on the artery by perfusion. In particular, the amount of elastic fibers (elastic fiber ratio) was highest in the internal carotid immediately after the bifurcation, which may reflect that the internal diameter of the internal carotid is approximately $1.5 \mathrm{~mm}$ 
narrower than that of the common carotid ${ }^{1)}$, so that the amount of elastic fibers in the tunica media (the elastic fiber ratio) may be greater because its structure is adapted to mitigate increased perfusion-related stress. It may also be affected by the tunica media, as this area is thinner than the proximal areas.

\section{Acknowledgement}

The authors declare no conflicts of interest associated with this manuscript.

\section{References}

1) Goto N, Ezure H: Chapter 1. Vascular Structure and Pathology. 1. Systemic Vascular Anatomy and Histology. Mizutani T, Kojima H., eds.: Supplement: The Progress of Medicine. Frontline of Aneu- rysms and Aneurysm Dissection. Tokyo: Ishiyaku Publishers, Inc. 2001, 3-12 [In Japanese].

2) Janzen J, Lanzer P, Rothernberger-Janzen K, Vuong PN: Variable extension of the transitional zone in the medial structure of carotid artery tripod. Vasa 2001; 30:101-106.

3) Hori E, Hayashi N, Masukoka T, Sato H, Kuwayama N, Hirashima Y, Endo S: Correlation of plaque localization with characteristics of the cervical carotid artery wall. Surg Cereb Stroke 2005; 33: 35-38 [In Japanese].

4) Kato Y, Mizutani T, Iizuka K, Kubo M, Sakamoto Y, Otsuka N, Ezure H, Inoue Y: Microscopic quantification of elastic fiber in tunica media of the human artery using photoshop. Showa Igaku. J Showa Med Soc 2016; 76:712-719 [in Japanese].

5) Henmi T: A statistical and histological study of the carotid and basilar arteries. J Nippon Med School 28 (3) 1961; 25:577-591 [In Japanese].

6) Masuda H, Kawamura K, Shozawa T: Elastic fibers in the media of the flow loaded canine carotid arteries. Journal of Japanese Society of Biorheology 1986; 1:19-26 [in Japanese]. 